Pacific Journal of Mathematics

INVARIANT SUBSPACE LATTICES FOR A CLASS OF
OPERATORS

Bom-hua ONG 


\section{INVARIANT SUBSPACE LATTICES FOR A CALSS OF OPERATORS}

\section{BOON-HUA ONG}

We study the invariant subspace lattices for a one parameter family of operators $\left\{T_{\alpha}\right\}_{\alpha}$ on $L^{p}(0,1), \alpha$ a complex number, where

$$
T_{\alpha} f(x)=x f(x)+\alpha \int_{0}^{x} f(t) d t,
$$

and their adjoints $T_{\alpha}^{*}$,

$$
T_{\alpha}^{*} f(x)=x f(x)+\alpha \int_{x}^{1} f(t) d t .
$$

The closed invariant subspaces for $T_{\alpha}$ are in one-to-one correspondence with certain closed ideals of $\widetilde{\mathscr{R}}_{\alpha}$, where $\widetilde{\mathscr{R}}_{\alpha}$ is a Silov algebra with unit and in which the range $\mathscr{R}_{\alpha}$ of the Riemann Liouville operator $J_{\alpha}$

$$
\left(J_{\alpha} f(x)=\frac{1}{\Gamma(\alpha)} \int_{0}^{x}(x-t)^{\alpha-1} f(t) d t\right)
$$

is embedded as a closed ideal. When $n$ is a positive integer, there is a complete lattice isomorphism between the closed ideals of $\widetilde{\mathscr{R}}_{n}$ and the $n$-tuples $\left(E_{0}, E_{1}, \cdots, E_{n-1}\right)$ of closed subsets of $[0,1]$ where $E_{0} \supseteq E_{1} \supseteq \cdots \supseteq E_{n-1} \supseteq$ derived set of $E_{0}$. Every closed ideal of $\widetilde{\mathscr{R}}_{n}$ is the intersection of closed primary ideals. Similar results carry over to $\alpha$ where the real part of $\alpha$ is an integer and also to the adjoint operators.

1. Introduction. Not many operators have had their invariants subspace lattices completely described. To name but a few, the invariant subspace lattice for the (simple) Volterra operator on $L^{p}(0,1)$ was completely determined by Donoghue [4] and a more general result by Kalisch [7], that for the (simple) shift operator on $\mathfrak{l}^{2}$ by Beurling [1]. Further investigation of the invariant subspaces for the weighted shift operators have been made by Donoghue [4], Korenbljum [12], Nikol'skii ([14], [15]) and many others.

The main object of this paper is to characterize the invariant subspace lattices for a one parameter family of operators $\left\{T_{\alpha}\right\}_{\alpha}$ on $L^{p}(0,1)$ (in general $1<p<\infty$, but in some cases $1 \leqq p<\infty$ ) where

$$
T_{\alpha} f(x)=x f(x)+\alpha \int_{0}^{x} f(t) d t
$$

$f \in L^{p}(0,1), x \in[0,1]$ and $\alpha$ is any complex number with integer real part. (And hence for their adjoints, namely $\left\{T_{\alpha}^{*}\right\}$ where $T_{\alpha}^{*} f(x)=$ 
$\left.x f(x)+\alpha \int_{x}^{1} f(t) d t.\right)$

We convert the invariant subspace problem into an equivalent problem where we characterize the closed ideals of certain Silov algebras. Sarason [18] had employed this approach to characterize all the closed invariant subspaces of $T_{1}$. Our result is a generalization of his. The equivalence of the two problems is established by using analysis of Kantorovitz [10] on the functional calculus of the operators $T_{\alpha}$.

We prove that there is a complete lattice isomorphism between the closed invariant subspaces of $T_{n}(n \in N$, the natural numbers) and certain closed ideals of a Silov algebra $\widetilde{\mathscr{R}}_{n}$ where $\widetilde{\mathscr{R}}_{n}=\left\{f: f^{(n-1)}\right.$ is absolutely continuous, $\left.f^{(n)} \in L^{p}(0,1)\right\}$ with norm $|f|_{n}=\left\|f^{(n)}\right\|_{p}+$ $\sum_{i=0}^{n-1}\left|f^{(i)}(0)\right|$ (Theorem 4.1). This correspondence is induced by the Riemann Liouville operator $J_{n}$ on $L^{p}(0,1)$ where

$$
\left.J_{\alpha} f(x)=\frac{1}{\Gamma(\alpha)} \int_{0}^{x}(x-t)^{\alpha-1} f(t) d t, \operatorname{Re} \alpha \text { (the real part of } \alpha\right)>0,
$$

$\Gamma$ is the gamma function.

There is a complete lattice isomorphism between the closed ideals of $\tilde{\mathscr{R}}_{n}$ and the $n$-tuples $\left(E_{0}, E_{1}, \cdots, E_{n-1}\right)$ of closed subsets of $[0,1]$ with $E_{0} \supseteqq E_{1} \supseteqq \cdots \supseteqq E_{n-1} \supseteqq$ derived set of $E_{0}$ (Theorems 3.19 and 4.3). Every closed ideal of $\widetilde{\mathscr{R}_{n}}$ is found to be the intersection of closed primary ideals. Several other algebras were known to have this property. Stone [21] proved it for the algebra $C[0,1]$, Silov [19] for $C^{1}[0,1]$, Whitney [24] for $C^{n}[0,1]$, Snol [20] for some algebras lying between $C[0,1]$ and $C^{1}[0,1]$, Osadchii [16] for the algebra of functions on the unit circle for which the $n$th derivatives are square summable, Daly and Downum [3] for a subalgebra of $C^{n-1}[0,1]$ consisting of functions whose $(n-1)$ th derivatives satisfy a bounded Lipschitz condition.

Similar results carry over easily to the more general parameter $\alpha$, where $\operatorname{Re} \alpha$ is an integer, and also the adjoints of these operators.

When $\operatorname{Re} \alpha$ is not integral, the situation is more complex. It is not apparent that $\mathscr{R}_{\alpha}$, the range of $J_{\alpha}$ is an algebra. Via functional calculus, we show that indeed it is, for $\operatorname{Re} \alpha \geqq 1$. Moreover it can be embedded as a closed ideal of a Silov algebra with unit, $\widetilde{\mathscr{R}}_{\alpha}$, which is a natural generalization of $\tilde{\mathscr{R}}_{n}$. As is in the case of $T_{n}$, there is a one-to-one correspondence between the closed $T_{\alpha}$-invariant subspaces and certain closed ideals of $\mathscr{R}_{\alpha}$ (and hence of $\widetilde{\mathscr{R}}_{\alpha}$ ). We conjecture that all the closed ideals of $\tilde{\mathscr{R}}_{\alpha}(n<\operatorname{Re} \alpha<n+1)$ are completely determined as in the case of $\tilde{\mathscr{R}}_{n}$, by $n$-tuples of closed subsets of $[0,1]$ satisfying certain conditions. We have not succeeded 
in proving this and we hope to return to the problem on a later occasion. Kantorovitz ([10], [11]) showed that the operator $T_{\alpha}$ has $C^{n}$-functional calculus if and only if $|\operatorname{Re} \alpha| \leqq n$. Imitating his argument, we found that for $\alpha, \beta \in C$ (the complex numbers) with $\operatorname{Re} \alpha \geqq$ 1 and $0 \leqq \operatorname{Re} \beta \leqq \operatorname{Re} \alpha, T_{\beta}$ has $\widetilde{\mathscr{R}}_{\alpha}$-functional calculus.

Finally it should be remarked that Erdös [5] and Waterman [23] had independently found all the invariant subspaces for the operator $T_{f}$ on $L^{p}(0,1)$ where $T_{f}$ is defined as

$$
T_{f} g(x)=f(x) g(x)-\int_{0}^{x} f^{\prime}(t) g(t) d t,
$$

$g \in L^{p}(0,1), x \in[0,1]$ and $f$ is a function with some suitable conditions. The particular case when $f(t)=t$ gives $T_{f}=T_{-1}$. Furthermore, Waterman, in [23], claims to have found all the closed invariant subspaces for the operators $\left\{T_{-n}\right\}, n$ a positive integer, by using recent results on $L^{p}$-approximation by splines. However, our work is conducted independently of his work which was not available to us $^{1}$.

2. The Silov algebra $\mathscr{R}_{n}$. The range $\mathscr{R}_{n}$ of the Riemann Liouville operator $J_{n}$,

$$
J_{n} f(x)=\frac{1}{\Gamma(n)} \int_{0}^{x}(x-t)^{n-1} f(t) d t, f \in L^{p}(0,1), x \in[0,1],
$$

where $n \in N, 1 \leqq p<\infty$, is given by

$$
\begin{aligned}
\mathscr{R}_{n}= & \left\{g: g^{(n-1)} \text { is absolutely continuous, } g^{(n)} \in L^{p}(0,1),\right. \\
& \left.g^{(i)}(0)=0,0 \leqq i \leqq n-1\right\} .
\end{aligned}
$$

Clearly $\mathscr{R}_{n}$ is a subalgebra of the well known Banach algebra $C^{n-1}[0,1]$, the space of all complex valued functions with $(n-1)$ continuous derivatives. But we will endow $\mathscr{R}_{n}$ with its own norm. As $\mathscr{R}_{n}$ has no unit, it is convenient to embed it in a larger algebra $\widetilde{\mathscr{R}}_{n}$ with unit, namely

$$
\tilde{\mathscr{R}}_{n}=\mathscr{R}_{n} \oplus \boldsymbol{C} x^{n-1} \oplus \boldsymbol{C} x^{n-2} \oplus \cdots \oplus \boldsymbol{C} x \oplus \boldsymbol{C}
$$

where the sum is direct. Thus

$$
\widetilde{\mathscr{R}}_{n}=\left\{g: g^{(n-1)} \text { is absolutely continuous, } g^{(n)} \in L^{p}(0,1)\right\} \text {. }
$$

Define a norm $|\cdot|_{n}$ on $\widetilde{\mathscr{R}}_{n}$ as follows: for $g \in \widetilde{\mathscr{R}}_{n}$,

$$
|g|_{n}=\left\|g^{(n)}\right\|_{p}+\sum_{i=0}^{n-1}\left|g^{(i)}(0)\right|,
$$

${ }^{1}$ Still unable to locate this reference. 
where $\|\cdot\|_{p}$ denotes the $L^{p}$-norm and $|\cdot|$ the absolute value. It is easily seen that $\left(\tilde{\mathscr{R}}_{n},|\cdot|_{n}\right)$ is complete and $\mathscr{R}_{n}$ is a closed ideal of $\widetilde{\mathscr{R}}_{n}$. The norm $\|\cdot \cdot\| \|_{n}$ defined as

$$
\|\| g\left\|_{n}=\right\| g^{(n)}\left\|_{p}+\sum_{i=1}^{n-1}\right\| g^{(i)} \|_{\infty}, \quad g \in \widetilde{\mathscr{R}}_{n},
$$

(where $\|\cdot\|_{\infty}$ is the supremum norm) is equivalent to $|\cdot|_{n}$.

Since for any $g, h \in \mathscr{\mathscr { R }}_{n},|h g|_{n} \leqq c|h|_{n}|g|_{n}$ for some constant $c$ independent of $g, h$ and $n, \widetilde{\mathscr{R}}_{n}$ can be made into a Banach algebra by the equivalent norm $|\cdot|_{n}^{\prime}$ where $|g|_{n}^{\prime}=c|g|_{n}$. Moreover $\tilde{\mathscr{R}}_{n}$ is a Silov algebra. (A commutative semisimple Banach algebra $\mathfrak{A}$ is a Silov algebra if for any closed subset $F$ of the maximal ideal space $\Phi_{a}$ of $\mathfrak{A}$ and $x \in \Phi_{a}, x \notin F$, there is an element $h \in \mathfrak{A}$ such that $h(x)=$ 1 and $h(F)=\{0\}$.) Henceforth we shall use the norm $|\cdot|_{n}$ on $\tilde{\mathscr{R}}_{n}$, but the arguments used work for both norms.

Observe that for any fixed $i$ and $a, 0 \leqq i \leqq n-1, a \in[0,1]$, the evaluation map $E_{i, a}: \tilde{\mathscr{R}}_{n} \rightarrow C$ defined as $E_{i, a}(g)=g^{(i)}(a), g \in \widetilde{\mathscr{R}}_{n}$, is continuous.

It will be seen that the collection $\mathscr{C}_{o}^{n}$ of all the closed ideals of $\mathscr{R}_{n}$ which are closed under multiplication by the function $x$ are in one-to-one correspondence with the closed invariant subspaces of $T_{n}$. $\quad \mathscr{C}_{0}^{n}$ consists of precisely those closed ideals of $\widetilde{\mathscr{R}}_{n}$ which lie in $\mathscr{R}_{n}$. The collection $\mathscr{C}^{n}$ of all the closed ideals of $\widetilde{\mathscr{R}}_{n}$ (and hence $\mathscr{C}_{0}^{n}$ ) can be neatly characterized and every closed ideal of $\widetilde{\mathscr{R}}_{n}$ is the intersection of closed primary ideals.

3. The MaIn Theorem. Characterization of the closed ideals of $\tilde{\mathscr{R}}_{n}$.

For any closed ideal $\mathscr{I}$ in $\tilde{\mathscr{R}_{n}}$, we define

$$
\begin{aligned}
& Z_{0}(\mathscr{F})=\text { Hull } \mathscr{F} \text {, and for } 0 \leqq i \leqq n-1, \\
& Z_{i}(\mathscr{F})=\left\{x \in[0,1]: f^{(j)}(x)=0, \forall 0 \leqq j \leqq i, \forall f \in \mathscr{I}\right\}
\end{aligned}
$$

Whenever there is no confusion, we abbreviate $Z_{i}(\mathscr{I})$ as $Z_{i}, 0 \leqq i \leqq$ $n-1$.

REMARK 3.1. For any closed ideal $\mathscr{I}$ in $\tilde{\mathscr{R}}_{n}$, deriv $Z_{0} \subseteq Z_{n-1} \subseteq$ $Z_{n-2} \subseteq \cdots \subseteq Z_{0}$, where deriv $Z_{0}$ is the derived set of $Z_{0}$.

Each closed ideal $\mathscr{F}$ not only determines an $n$-tuple of sets $Z_{0}$, $Z_{1}, \cdots, Z_{n-1}$, but in fact is completely determined by these sets. We shall now state the main theorem.

MAIN Theorem 3.2. For any $n$-tuple of closed subsets $\left(E_{0}, E_{1}, \cdots\right.$, 
$\left.E_{n-1}\right)$ with deriv $E_{0} \subseteq E_{n-1} \subseteq E_{n-2} \subseteq \cdots \subseteq E_{0} \subseteq[0,1]$, there exists a unique closed ideal $\mathscr{I}$ in $\widetilde{\mathscr{R}}_{n}$ such that $Z_{i}=E_{i}, 0 \leqq i \leqq n-1$.

The following remark will be useful in establishing the uniqueness part of the main theorem.

REMARK 3.3. Let $E, F$ be any closed subset of $[0,1]$ such that $\operatorname{deriv} F \subseteq E \subseteq F$. Then $F \backslash E$ consists of at most countably many isolated points.

The existence of a closed ideal of $\widetilde{\mathscr{R}}_{n}$ satisfying the required properties is easily established. There is an obvious candidate.

THEOREM 3.4. Given $E_{0} \supseteq E_{1} \supseteqq \ldots \supseteqq E_{n-1}$, closed subsets of $[0,1]$ such that $E_{n-1} \supseteqq \operatorname{deriv} E_{0}$, there exists a closed ideal $\mathscr{I}$ in $\widetilde{\mathscr{R}}_{n}$ such that $Z_{i}=E_{i}, 0 \leqq i \leqq n-1$.

Proof. $\mathscr{I}=\left\{f \in \widetilde{\mathscr{R}}_{n}: f=f^{(1)}=\cdots=f^{(i)}=0\right.$ on $E_{i}, 0 \leqq i \leqq$ $n-1\}$, has all the required properties.

There are some special ideals which play an important role in the proof of uniqueness. For any $n$-tuple $\left(E_{0}, E_{1}, \cdots, E_{n-1}\right)$ of closed subsets of $[0,1]$ such that deriv $E_{0} \subseteq E_{n-1} \subseteq E_{n-2} \subseteq \cdots \subseteq E_{0}$, define

$$
\begin{aligned}
\mathscr{C}\left(E_{0}, E_{1}, \cdots, E_{n-1}\right)= & \left\{f \in \tilde{\mathscr{R}}_{n}: f=f^{(1)}=\cdots=f^{(i)}=0\right. \\
& \text { on } \left.E_{i}, 0 \leqq i \leqq n-1\right\} .
\end{aligned}
$$

We shall abbreviate $\mathscr{C}(E, E, \cdots, E)$ by $\mathscr{C}(E)$. Let

$$
\begin{aligned}
\mathscr{L}_{0}\left(E_{0}, E_{1}, \cdots, E_{n-1}\right)= & \left\{f \in \mathscr{C}\left(E_{0}, E_{1}, \cdots, E_{n-1}\right): f=0\right. \\
& \text { in some neighborhood of } \left.E_{n-1}\right\},
\end{aligned}
$$

and for any closed set $F$ in $[0,1]$,

$\mathscr{J}(F)=\left\{f \in \widetilde{\mathscr{R}}_{n}: f=0\right.$ in some neighborhood of $\left.F\right\}$.

$\mathscr{M}\left(E_{0}, E_{1}, \cdots, E_{n-1}\right)$ is clearly a closed ideal but $\mathscr{M}_{0}\left(E_{0}, E_{1}, \cdots, E_{n-1}\right)$

and $\mathscr{J}(\boldsymbol{F})$ are ideals which may not be closed.

Two general results quoted below will be useful.

TheOREm 3.5. ([13], p. 225, Thm. 4). Let $\mathfrak{A}$ be a Silov algebra and $F$ a closed subset of the maximal ideal space of $\mathfrak{A}$. Let $\mathscr{J}$ be an ideal such that hull $\mathscr{I}=F$. Then $\mathscr{J}(F) \leqq \mathscr{I}$ and hull $\mathscr{J}(F)=F$.

In other words, $\mathscr{J}(F)$ is the smallest ideal with hull $F$. 
Proposition 3.6. Let $X$ be a Banach space and $Y$ a dense subspace of $X$. If $M$ is a closed subspace of $X$ which is of finite codimension, then $M \cap Y$ is dense in $M$.

Briefly, the process of establishing that every closed ideal of $\widetilde{\mathscr{R}}_{n}$ is uniquely determined by the closed subset $Z_{i}, 0 \leqq i \leqq n-1$, consists of three major steps. First we analyze the structure of the closure $\overline{\mathscr{J}(F)}$ of $\mathscr{J}(F)$ where $F$ is any closed subset of $[0,1]$. We then prove that $\mathscr{L}_{0}\left(Z_{0}(\mathscr{F}), \cdots, Z_{n-1}(\mathscr{F})\right) \subseteq \mathscr{J}$ and lastly we prove $\mathscr{L}_{0}\left(E_{0}, \cdots, E_{n-1}\right)=\mathscr{C}\left(E_{0}, \cdots, E_{n-1}\right)$. It is immediate from the last two steps that $\mathscr{F}=\mathscr{\mathscr { C }}_{0}\left(Z_{0}(\mathscr{I}), \cdots, Z_{n-1}(\mathscr{I})\right)=\mathscr{M}\left(Z_{0}(\mathscr{I}), \cdots\right.$, $\left.Z_{n-1}(\mathscr{J})\right)$.

Proposition 3.7. In $\tilde{\mathscr{R}}_{n}, \overline{\mathscr{J}(\{\lambda\})}=\mathscr{C l}(\{\lambda\}), \lambda \in[0,1]$.

Proof. Clearly $\overline{\mathscr{J}(\{\lambda\})} \cong \mathscr{M}(\{\lambda\})$, so it suffices to show $\mathscr{M}(\{\lambda\}) \cong$ $\bar{J}(\{\lambda\})$.

Let $f \in \mathscr{C}(\{\lambda\})$, then $f^{(i)}(\lambda)=0,0 \leqq i \leqq n-1$. Define

$$
K_{m}=[0,1] \backslash\left[\lambda-\frac{1}{m}, \lambda+\frac{1}{m}\right]
$$

and

$$
f_{m}(x)=\int_{\lambda}^{x} \int_{\lambda}^{r_{1}} \cdots \int_{\lambda}^{r_{n-1}} f^{(n)}(t) \chi_{K_{m}}(t) d t d r_{n-1} \cdots d r_{1},
$$

where $m$ is a positive integer. Then $f_{m} \in \mathscr{J}(\{\lambda\})$ and $f_{m} \rightarrow f$ in $\tilde{\mathscr{R}}_{n}$, since $f_{m}^{(j)}(t) \rightarrow f^{(j)}(t)$ uniformly for $0 \leqq j \leqq n-1$, and $f_{m}^{(n)} \rightarrow f^{(n)}$ in $L^{p}(0,1)$. Thus $f \in \overline{\mathscr{J}(\{\lambda\})}$. This completes the proof.

As a consequence, the closed primary ideals of $\tilde{\mathscr{R}}_{n}$ are easily identified. (An ideal is primary if it is contained in a unique maximal ideal.) Indeed, they have simple structures.

COROLLARY 3.8. Any closed primary ideal $\mathscr{F}$ of $\tilde{\mathscr{R}}_{n}$ is of the form $\mathscr{M}(\{\lambda\},\{\lambda\}, \cdots,\{\lambda\}, \phi, \cdots, \phi)$ where $\{\lambda\}=$ Hull $\mathscr{F}$ and the multiplicity of $\lambda$ whithin the parenthesis is $i+1$ for $0 \leqq i \leqq n-1$.

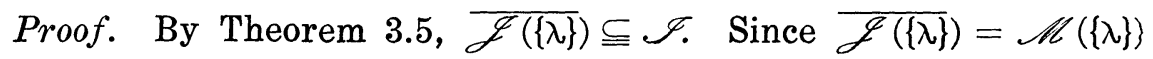
and $\mathscr{M}(\{\lambda\})$ has finite codimension, it follows that $\mathscr{J}$ also has finite codimension. Thus $\mathscr{P} \cap \mathscr{F}$ is dense in $\mathscr{A}$, since the set $\mathscr{P}$ of all polynomials is dense in $\tilde{\mathscr{R}}_{n}$. This implies that Hull $(\mathscr{P} \cap \mathscr{I})=$ Hull $\mathscr{F}$. Further-more $\mathscr{P} \cap \mathscr{F}$ is an ideal in $\mathscr{P}$ and thus is a principal ideal whose generator must be of the form $(x-\lambda)^{i+1}$ for some $0 \leqq i \leqq n-1$. 
Hence $\mathscr{I}=\left\{f \in \tilde{\mathscr{R}}_{n}: f(\lambda)=\cdots=f^{(i)}(\lambda)=0\right\}$, that is $\mathscr{I}=\mathscr{C}(\{\lambda\}$, $\cdots,\{\lambda\}, \phi, \cdots, \phi)$.

REMARK 3.9. The argument used in Proposition 3.7 still holds when the point $\lambda$ is replaced by an interval $[a, b] \subseteq[0,1]$.

LEMMA 3.10. Let $F_{1}, F_{2}$ be disjoint closed subsets of the maximal ideal space of a Silov algebra $\mathfrak{A}$. Let $\mathscr{F}_{1}, \mathscr{F}_{2}$ be closed ideals with $\overline{\mathscr{J}\left(F_{i}\right)}=\mathscr{I}_{i}, i=1,2$. Then $\overline{\mathscr{J}\left(F_{1} \cup F_{2}\right)}=\mathscr{I}_{1} \cap \mathscr{I}_{2}$.

Proof. Clearly $\overline{\mathscr{J}\left(F_{1} \cup F_{2}\right)} \cong \overline{\mathscr{J}\left(F_{1}\right)} \cap \overline{\mathscr{J}\left(F_{2}\right)}=\mathscr{F}_{1} \cap \mathscr{F}_{2}$. To show the reverse inclusion, let $f \in \mathscr{F}_{1} \cap \mathscr{F}_{2}$. Choose $g_{i} \in \mathfrak{A}, i=1,2$ such that

$$
g_{1}= \begin{cases}f & \text { in a neighborhood of } F_{1} \\ 1 & \text { in a neighborhood of } F_{2}\end{cases}
$$

and

$$
g_{2}=\left\{\begin{array}{lll}
f & \text { in a neighborhood of } F_{2} \\
1 & \text { in a neighborhood of } F_{1}
\end{array}\right.
$$

Then $f=g_{1} g_{2}+h$ where $h \in \mathscr{J}\left(F_{1} \cup F_{2}\right.$ ). Observe that $g_{i} \in \mathscr{J}_{i}$ (by a property of Silov algebras ([13], p. 224, Thm. $\left.3^{\prime \prime}\right)$ ), so we can choose $U_{k, i} \in \mathscr{J}\left(F_{i}\right)$ such that $U_{k, i} \rightarrow g_{i}$ as $k \rightarrow \infty$. Then $U_{k, 1} U_{k, 2}+h \rightarrow f$. Moreover, $U_{k, 1} U_{k, 2}+h \in \mathscr{J}\left(F_{1} \cup F_{2}\right)$, thus $\left.f \in \overline{\mathscr{J}\left(F_{1} \cup F_{2}\right.}\right)$.

REMARK 3.11. The above result, which holds for a general Silov algebra, enables us to conclude that, in $\tilde{\mathscr{R}}_{n}, \overline{\mathcal{J}(F)}=\mathscr{L}(F)$ for any closed $F$ which is a finite disjoint union of points and closed intervals.

The following observation will be used.

Observation 3.12. Let $\left\{\left[a_{i}, b_{i}\right]\right\}$ be a countable disjoint collection of intervals in $[0,1]$ such that $f, f^{(1)}, \cdots, f^{(n-1)}$ vanish at all the $a_{i}$ 's and $b_{i}$ 's. Then clearly the truncated function $f \chi_{[0,1] \cup \cup_{i}\left[a_{i}, b_{i}\right]} \in \widetilde{\mathscr{R}}_{n}$, where $\chi_{E}$ is the characteristic function on $E$.

We can now describe $\overline{\mathscr{J}(F)}$ for any closed subset $F \cong[0,1]$, for this general case can be reduced to the situation of Remark 3.11.

THeOREM 3.13. In $\tilde{\mathscr{R}}_{n}, \overline{\mathscr{J}(F)}=\mathscr{C}(F)$ for any closed $F \subseteq[0,1]$.

Proof. We only need to show $\mathscr{C}(F) \subseteq \overline{\mathscr{J}(F)}$. Let $f \in \mathscr{M}(F)$. The set $F^{c}$, the complement of $F$ in $[0,1]$, being open, is a disjoint 
union of a countable number of open intervals (with the possible exception that the interval with end point 0 or 1 might be closed at that end). Let $F^{c}=\bigcup_{i=1}^{\infty}\left(a_{i}, b_{i}\right)$ (the union may be finite). There exists $N>0$ such that $\left\|f^{(n)} \chi_{U_{i}>N\left(a_{i}, b_{i}\right)}\right\|_{p}$ is very small. Let $G=\bigcup_{i=1}^{N}\left(a_{i}, b_{i}\right)$. (Here $G$ includes the interval with end point 0 if there is one.) Define $\tilde{f}=f \chi_{G}$; then by Remark $3.11 \tilde{f} \in \overline{\mathcal{J}\left(G^{c}\right)} \cong$ $\overline{\mathscr{J}(F)}$ and it approximates $f$, therefore $f \in \overline{\mathscr{J}(F)}$ and we have the result.

COROLLARY 3.14. Let $\mathscr{I}$ be a closed ideal of $\tilde{\mathscr{R}}_{n}$, then $\mathscr{I} \supseteqq$ $\mathscr{l}\left(Z_{0}(\mathscr{J})\right)$.

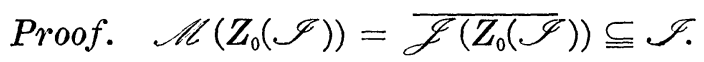

LEMMA 3.15. Let $\mathscr{I}$ be a closed ideal of $\widetilde{\mathscr{R}}_{n}$ and let $h \in \mathscr{I}$ be such that $h^{(i)}(a) \neq 0$ for some $0 \leqq i<n$ and some $a \in[0,1]$. Then for any neighborhood $N_{a}$ of a, there exists $H \in \mathscr{I}$ such that support $(H) \subseteq N_{a}$ and $H^{(l)}(a)=0$ for $0 \leqq l<n, l \neq i$, but $H^{(i)}(a) \neq 0$.

Proof. Let $k$ be the smallest positive integer $\leqq i$ such that $h^{(k)}(a) \neq 0$. Define

$$
Q(x)=(x-a)^{i-k} \cdot h(x)
$$

and $g(x)=Q(x) \cdot\left(1+\sum_{n=1}^{(n-1)-i} C_{j} \cdot(x-a)^{j}\right)$, where the $C_{j}$ 's are constants yet to be determined. For $\mathfrak{l}<i, g^{(i)}(a)=0$ while $g^{(i)}(a) \neq 0$. The fact that $Q^{(i)}(a) \neq 0$ and $Q^{m}(a)=0$ for $m<i$, enables the $C_{j}$ 's to be suitably chosen successively so as to make $g^{(\mathfrak{l})}(a)=0$ for $i<\mathfrak{l}<n$ while $g^{(1)}(a)$ remains unchanged for $\mathfrak{l} \leqq i$. Let $f$ be a $C^{\infty}$ function on $[0,1]$ such that support $(f) \subseteq N_{a}$ and $f=1$ in some neighborhood of $a$. Evidently $H=g f$ has all the required properties.

For any arbitrary $f$ in any ideal $\mathscr{I}$ of $\tilde{\mathscr{R}}_{n}$, the above lemma annihilate its derivative (of any order $\leqq n-1$ ) at any point $a \in[0,1]$ by the addition of some appropriate function in $\mathscr{F}$ but simultaneously leaving all the other derivatives at a undisturbed.

LEMMA 3.16. For any closed ideal $\mathscr{J}$ of $\tilde{\mathscr{R}}_{n}, \mathscr{J} \supseteqq \mathscr{L}_{0}\left(Z_{0}(\mathscr{I})\right.$, $\left.\cdots, Z_{n}(\mathscr{I})\right)$.

Proof. Let $f \in \mathscr{L}_{0}\left(Z_{0}(\mathscr{F}), \cdots, Z_{n-1}(\mathscr{F})\right)$; then $f=0$ in some neighborhood $U \supseteq Z_{n-1}(\mathscr{J})$. Since $Z_{n-1}(\mathscr{J}) \supseteqq \operatorname{deriv} Z_{0}(\mathscr{I})$, the closed sets $Z_{j}(\mathscr{I}) \backslash U$ must be finite for all $0 \leqq j \leqq n-2$. Using Lemma 3.15 , repeatedly if necessary, we can find $G \in \mathscr{J}$ such that the function $K=f+G$ has the property that $K^{(i)}$ vanishes on 
$Z_{0}(\mathscr{J}), 0 \leqq i \leqq n-1$. Thus $K \in \mathscr{J}$ by Corollary 3.14 , and hence $f \in \mathscr{F}$.

The simple well behaved function given below is worth noting, for it makes the calculation on annihilation work very neatly.

LEMMA 3.17. Let

$$
Q(x)=\int_{0}^{x} \int_{0}^{r_{1}} \cdots \int_{0}^{r_{n-1}} \chi_{[\alpha, \beta]} d t d r_{n-1} \cdots d r_{1},
$$

where $[\alpha, \beta] \cong[0,1]$. Then

$$
Q(x)=0 \text { for } \quad x \leqq \alpha
$$

and

$$
Q^{(i)}(x)=\frac{(x-\alpha)^{n-i}}{(n-i) !} \text { for } \alpha \leqq x \leqq \beta, \quad 0 \leqq i \leqq n-1
$$

In the process of establishing $\overline{\mathscr{L}_{0}\left(E_{0}, \cdots, E_{n-1}\right)}=\mathscr{C}\left(E_{0}, \cdots, E_{n-1}\right)$, several reductions occur but the chief and final burden is shouldered by the miniature case described in the main lemma.

MAIN Lemma 3.18. Let

$$
K(x)=\int_{0}^{x} \int_{0}^{r_{1}} \cdots \int_{0}^{r_{n-1}} f(t) d t d r_{n-1} \cdots d r_{1},
$$

where $f \in L^{p}(0,1)$. Suppose $\left\{x_{j}\right\}_{1}^{\infty}$ is a sequence of distinct isolated zeros of $K$ such that $x_{1}<x_{2}<\cdots$ and $x_{j} \rightarrow 1$. Given $\varepsilon>0$, there exist consecutive points $a, b \in\left\{x_{j}\right\}_{1}^{\infty}$ and a function $G$ such that

$$
\begin{array}{lll}
G(x)=K(x) & \text { for } & x \leqq a \\
G(x)=0 & \text { for } & b \leqq x \leqq 1
\end{array}
$$

and

$$
|K-G|_{n}<\varepsilon
$$

Proof. We may assume $K$ to be a real function (for otherwise we can write $K$ as $K_{1}+i K_{2}$ with $K_{1}, K_{2}$ real). Given $\varepsilon>0$, there exist $a_{1}, a_{2}, \cdots, a_{n} \in\left\{x_{j}\right\}_{1}^{\infty}$ such that $0<a_{1}<a_{2}<\cdots<a_{n}<1$, the $a_{i}$ 's are consecutive points in $\left\{x_{j}\right\}_{1}^{\infty}$, and $\left\|K^{(n)} \chi_{\left[a_{1}, 1\right]}\right\|_{p}<\varepsilon$.

Denote the largest interval of $\left\{\left[a_{1}, a_{2}\right],\left[a_{2}, a_{3}\right], \cdots,\left[a_{n-1}, a_{n}\right]\right\}$ by $[a, b]$. Since $K\left(a_{1}\right)=K\left(a_{2}\right)=\cdots=K\left(a_{n}\right)=0$, there exists $t_{j} \in\left(a_{1}, a_{n}\right)$ such that $K^{i}\left(t_{j}\right)=0,1 \leqq j \leqq n-1$.

$$
\begin{aligned}
\left|K^{(n-1)}(b)\right| & =\left|\int_{t_{n-1}}^{b} K^{(n)}(s) d s\right| \\
& \leqq \varepsilon[(n-1)(b-a)]^{1 / q} \quad(\text { for } p>1) .
\end{aligned}
$$


Similarly $\left|K^{(j)}(b)\right| \leqq \varepsilon[(n-1)(b-a)]^{(n-1)-j+1 / q}, 0 \leqq j \leqq n-2$. When $p=1$, the estimates are

$$
\left|K^{(j)}(b)\right| \leqq \varepsilon[(n-1)(b-a)]^{(n-1)-j}, \quad 0 \leqq j \leqq n-1 .
$$

We shall confine ourselves to the case $p>1$, for the same argument works for the case $p=1$. Denote the length of the interval $[a, b]$ by $n \delta, \delta>0$. Let $s_{1}, s_{2}, \cdots, s_{n}$ be $n$ equally spaced points in $[a, b]$ such that $s_{1}>s_{2}>\cdots>s_{n}=a$ and $b-s_{j}=j \delta, 1 \leqq j \leqq n$. Let

$$
\begin{aligned}
& L(x)=K(x)-\sum_{j=1}^{n} Q_{j}(x), \text { where } \\
& \qquad Q_{j}(x)=\int_{0}^{x} \int_{0}^{r_{1}} \cdots \int_{0}^{r_{n-1}} C_{j} \chi_{\left[s_{j}, b\right]}(t) d t d r_{n-1} \cdots d r_{1},
\end{aligned}
$$

and where the $C_{j}$ 's are constants yet to be determined.

Let $G=L \chi_{[0, b]}$. We need $L(b)=L^{(1)}(b)=\cdots=L^{(n-1)}(b)=0$ so that $G \in \tilde{\mathscr{R}}_{n}$ and we want $|K-G|_{n}<\varepsilon$. Now

$$
|K-G|_{n} \leqq\left\|K^{(n)} \chi_{\left[a_{1}, 1\right]}\right\|_{p}+\sum_{j=1}^{n}\left|Q_{j}\right|_{n}
$$

and

$$
\left|Q_{j}\right|_{n}=\left|C_{j}\right|\left(b-s_{j}\right)^{1 / p}=\left|C_{j}\right|(j \delta)^{1 / p} .
$$

Observe that $L(x)=K(x)$ for $x \leqq a$,

$$
\begin{aligned}
& L(b)=-\sum_{j=1}^{n} \frac{C_{j}(j \delta)^{n}}{n !} \\
& L^{(i)}(b)=K^{(i)}(b)-\sum_{j=1}^{n} \frac{C_{j}(j \delta)^{n-i}}{(n-i) !}, \quad 1 \leqq i \leqq n-1 .
\end{aligned}
$$

Hence we require

$$
\left\{\begin{array}{l}
\sum_{j=1}^{n} C_{j}(j \delta)=K^{(n-1)}(b) \\
\sum_{j=1}^{n} C_{j}(j \delta)^{2}=2 K^{(n-2)}(b) \\
\vdots \\
\sum_{j=1}^{n} C_{j}(j \delta)^{n-1}=(n-1) ! K^{(1)}(b) \\
\sum_{j=1}^{n} C_{j}(j \delta)^{n}=0
\end{array}\right.
$$

and $\left|C_{j}\right|(j \delta)^{1 / p}<\varepsilon, 1 \leqq j \leqq n$. Treating the $C_{j}$ 's as unknowns, $\left(^{*}\right)$ is a system of simultaneous linear equations which can be solved by Cramer's rule. The determinant 


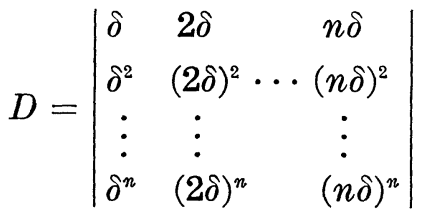

$$
\begin{aligned}
& =\delta(2 \delta) \cdots(n \delta) \prod_{1 \leqq k<j \leqq n}(k-j) \delta
\end{aligned}
$$

and

$$
\left|C_{1}\right|=\frac{1}{D}\left|\begin{array}{cccc}
K^{(n-1)}(b) & 2 \delta & \cdots & n \delta \\
2 K^{(n-2)}(b) & (2 \delta)^{2} & & (n \delta)^{2} \\
\vdots & \vdots & & \vdots \\
(n-1) ! K^{(1)}(b) & & & \\
0 & (2 \delta)^{n} & \cdots & (n \delta)^{n}
\end{array}\right|
$$

Since $K^{(j)}(b) \leqq \varepsilon[n(n-1) \delta]^{n-1-j+1 / q}, 0 \leqq j \leqq n-1$, it is clear that,

$$
\left|C_{1}\right| \leqq \frac{d_{1} \varepsilon \delta^{n(n+1) / 2-1+1 / q}}{\delta^{n(n+1) / 2}}
$$

where $d_{1}$ is a positive constant which depends only on $n$. Therefore $\left|C_{1}\right| \delta^{1 / p} \leqq d_{1} \varepsilon$. Similarly there exist constants $d_{j}>0$ such that

$$
\left|C_{j}\right|(j \delta)^{1 / p} \leqq d_{j} \varepsilon, \quad 2 \leqq j \leqq n,
$$

where the $d_{j}$ 's depend only on $n$ and are independent of the choice of $b$. Thus $|K-G|_{n}<B \varepsilon$, where $B$ is a constant dependent only on $n$.

We are now ready to prove that $\mathscr{I}$ is completely determined by the sets $Z_{0}(\mathscr{J}), Z_{1}(\mathscr{F}), \cdots, Z_{n-1}(\mathscr{F})$.

THeOREM 3.19. For any closed ideal $\mathscr{I}$ of $\widetilde{\mathscr{R}}_{n}, \mathscr{I}=\mathscr{M}\left(Z_{0}(\mathscr{I})\right.$, $\left.\left.\cdots, Z_{n-1}(\mathscr{I})\right)=\overline{\mathscr{C}_{0}\left(Z_{0}(\mathscr{I}), \cdots, Z_{n-1}(\mathscr{I})\right.}\right)$.

Proof. Clearly $\left.\overline{\mathscr{L}_{0}\left(Z_{0}(\mathscr{I}), \cdots, Z_{n-1}(\mathscr{I})\right.}\right) \subseteq \mathscr{I} \subseteq \mathscr{C}\left(Z_{0}(\mathscr{I}), \cdots\right.$, $\left.Z_{n-1}(\mathscr{I})\right)$. So it suffices to show $\mathscr{C}\left(Z_{0}(\mathscr{J}), \cdots, Z_{n-1}(\mathscr{I})\right) \subseteq \overline{\mathscr{L}_{0}\left(Z_{0}(\mathscr{I})\right.}$, $\cdots, Z_{n-1}(\mathscr{I})$.

Case A. $Z_{n-1}(\mathscr{I})=\phi$.

Since $Z_{n-1}(\mathscr{J})=\phi, \mathscr{C}\left(Z_{0}(\mathscr{I}), \cdots, Z_{n-1}(\mathscr{I})\right)=\mathscr{L}_{0}\left(Z_{0}(\mathscr{J}), \cdots\right.$, $\left.Z_{n-1}(\mathscr{I})\right)$ by definition. Hence $\mathscr{\mathscr { L }}_{0}\left(Z_{0}(\mathscr{I}), \cdots, Z_{n-1}(\mathscr{I})\right)=\mathscr{\mathscr { C }}\left(Z_{0}(\mathscr{J})\right.$, $\left.\cdots, Z_{n-1}(\mathscr{J})\right)$.

Case B. $Z_{n-1}(\mathscr{J}) \neq \varnothing$. 
Let $f \in \mathscr{M}\left(Z_{0}(\mathscr{I}), \cdots, Z_{n-1}(\mathscr{I})\right)$. Being open, $\left(Z_{n-1}(\mathscr{J})\right)^{c}=$ $\bigcup_{i=1}^{\infty}\left(a_{i}, b_{i}\right)$, a countable (possibly finite) disjoint union of open intervals. Given $\varepsilon>0$, there exists $N>0$ such that $\left\|f^{(n-1)} \chi_{U_{i>N}\left(a_{i}, b_{i}\right)}\right\|_{p}<\varepsilon$. Also $f \chi_{\cup_{1 \leqq i \leqq N}\left(a_{i}, b_{i}\right)} \in \tilde{\mathscr{R}}_{n}$. Let $f_{k}=f \chi_{\left[a_{k}, a_{k}\right]}, 1 \leqq k \leqq N$. It suffices to show that each of the functions $f_{k}$ lies in $\overline{\mathscr{C}_{0}\left(Z_{0}(\mathscr{I}), \cdots, Z_{n-1}(\mathscr{J})\right)}$. Note that each interval $\left(a_{k}, b_{k}\right)$ contains at most a countable subset of $Z_{0}(\mathscr{J})$ whose only possible limit points are end points $a_{k}, b_{k}$ (by Remark 3.3). By a linear transformation, we can assume without loss of generality that $a_{k}=0$ and $b_{k}=1$. Now there exists points $s, t \in[0,1]$ such that $0<s<t<1$ and $[s, t] \cap Z_{0}(\mathscr{I})=\phi$. We can find $C^{\infty}$ functions $y_{1}, y_{2}$ such that $y_{1}+y_{2}=1$ and $y_{1}$ is supported in $[0, t]$ while $y_{2}$ is supported in $[s, 1]$. Let $\phi_{1}=f_{k} y_{1}$ and $\phi_{2}=f_{k} y_{2} ;$ trivially $f_{k}=$ $\phi_{1}+\phi_{2}$. It suffices to show $\phi_{2} \in \overline{\mathscr{L}_{0}\left(Z_{0}(\mathscr{I}), \cdots, Z_{n-1}(\mathscr{I})\right)}$ for $\phi_{1}$ can be similarly dealt with by applying the affine transformation $x \rightarrow$ $1-x$. It should however be noted that for any $H \in \widetilde{\mathscr{R}}_{n}$, the composition function defined as $G(x)=H(1-x)$ also belongs to $\widetilde{\mathscr{R}}_{n}$ since $G^{(i)}(x)=(-1)^{i} H^{(i)}(1-x), 1 \leqq i \leqq n$.

For $\phi_{2}$, there are two subcases:

Subcase 1. $F_{0}=\left(Z_{0}(\mathscr{J}) \backslash Z_{n-1}(\mathscr{J})\right) \cap[t, 1]$ is finite. Let $F_{j}=$ $\left(Z_{j}(\mathscr{I}) \backslash Z_{n-1}(\mathscr{I})\right) \cap[t, 1], 1 \leqq j \leqq n-2$, and $F_{n-1}=Z_{n-1}(\mathscr{I}) \cap[t, 1]$, then $F_{j} \subseteq F_{0}, 1 \leqq j \leqq n-2$ and $F_{n-1} \leqq\{1\}$. Recall that by Theorem 3.13, $\mathscr{C}(Z)=\overline{\mathscr{J}(Z)}$ for any closed $Z \subseteq[0,1]$. Take $Z=[0, s] \cup$ $F_{n-1}$. Since $\mathscr{C}\left(F_{0}, F_{1}, \cdots, F_{n-2}, \phi\right)$ is of finite codimension in $\widetilde{\mathscr{R}}_{n}$ and is closed, then by Proposition 3.6, $\mathscr{J}(Z) \cap \mathscr{C}\left(F_{0}, \cdots, F_{n-2}, \phi\right)$ is dense in $\mathscr{M}\left(Z \cup F_{0}, \cdots, Z \cup F_{n-2}, Z\right)$. Thus $\phi_{2} \in \mathscr{\mathscr { L }}_{0}\left(Z_{0}(\mathscr{J}), \cdots\right.$,

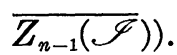

Subcase 2. $\quad F_{0}=\left(Z_{0}(\mathscr{F}) \backslash Z_{n-1}(\mathscr{I})\right) \cap[t, 1)=\left\{x_{1}, x_{2}, \cdots\right\}$, where $x_{i} \rightarrow 1$. Note that $Z_{n-1}(\mathscr{J}) \cap[t, 1]=\{1\} . \quad \phi_{2}(0)=\phi_{2}^{(1)}(0)=\cdots=\phi_{2}^{(n-1)}(0)=$ 0 , so $\phi_{2} \in \mathscr{R}_{n}$ and hence can be written in the form $\phi_{2}(x)=\int_{0}^{x} \int_{0}^{r_{1}} \cdots$ $\int_{0}^{r_{n-1}} f(t) d r_{n-1} \cdots d r_{1}$, for some $f \in L^{p}(0,1)$. The Main Lemma 3.18 implies that $\phi_{2} \in \overline{\mathscr{L}_{0}\left(Z^{0}(\mathscr{I}), \cdots, Z_{n-1}(\mathscr{I})\right)}$. This completes the proof.

CoROLLARY 3.20. Every closed ideal $\mathscr{F}$ in $\tilde{\mathscr{R}}_{n}$ is the intersection of closed primary ideals of $\mathscr{R}_{n}$.

Proof.

$$
\begin{aligned}
\mathscr{I} & =\mathscr{C}\left(Z_{0}(\mathscr{I}), \cdots, Z_{n-1}(\mathscr{I})\right) \\
& =\bigcap_{i=0}^{n-1} \bigcap_{\lambda \in Z_{i}(\mathcal{G})} \mathscr{L}(\{\lambda\},\{\lambda\}, \cdots,\{\lambda\}, \phi, \cdots, \phi)
\end{aligned}
$$

where the multiplicity of $\lambda$ within the parenthesis is $i+1$. 
REMARK 3.21. All the ideals in $\mathscr{C}_{0}^{n}$ are of the form $\mathscr{C}\left(E_{0}, \cdots\right.$, $\left.E_{n-1}\right)$ with $0 \in E_{i}, 0 \leqq i \leqq n-1$ and deriv $E_{0} \subseteq E_{n-1} \subseteq \cdots \leqq E_{0}$, where the $E_{i}$ 's are closed subsets of $[0,1]$.

4. Invariant subspaces of $T_{n}$ and closed ideals of $\tilde{\mathscr{R}}_{n}$ : Their correspondence and lattice structures. $J_{n}$ is a homeomorphism from $L^{p}(0,1)$ onto $\mathscr{R}_{n}$. Its bounded inverse, $J_{n}^{-1}$, is naturally the differentiation operator of order $n$ on $\mathscr{R}_{n}$, namely

$$
J_{n}^{-1} g(x)=\frac{d^{n}}{d x^{n}} g(x), g \in \mathscr{R}_{n}, x \in[0,1] .
$$

Moreover, by Leibnitz's rule and Fubini's theorem,

$$
J_{n}^{-1} M J_{n} f=T_{n} f \text { for } f \in L^{p}(0,1) \text {, where } M f(x)=x f(x), x \in[0,1] .
$$

This similarity relation achieves the following correspondance:

$\mathscr{S}$ is a closed $T_{n}$-invariant subspace of $L^{p}(0,1) \Leftrightarrow J_{n} \mathscr{S} \in \mathscr{C}_{0}^{n}$.

(From here onwards $\mathscr{C}_{0}^{n}$ will include the improper ideal $\mathscr{R}_{n}$ and the trivial ideal \{zero function\}, similarly for the collection $\mathscr{C}^{n}$.) We have thus proved the following result.

THEOREM 4.1. The map $J_{n}$ establishes a one-to-one correspondence between all the closed $T_{n}$-invariant subspaces and all the ideals in $\mathscr{C}_{0}^{n}$ via the relation $J_{n}^{-1} M J_{n}=T_{n}$. Thus the closed $T_{n}$-invariant subspaces are in one-to-one correspondence with the n-tuples $\left(E_{0}, E_{1}\right.$, $\left.\cdots, E_{n-1}\right)$ of closed subsets of $[0,1]$ where $E_{0} \supseteq E_{1} \supseteqq \cdots \supseteq E_{n-1} \supseteqq$ deriv $E_{0}$ and $0 \in E_{i}, 0 \leqq i \leqq n-1$.

Observation 4.2. We note an interesting observation that falls out immediately of our above discussion without further effort.

Let $T_{1}^{k}=T_{1} T_{1} \cdots T_{1}(k$ times, $k \in N)$, then $T_{1}^{k}=J_{1}^{-1} M\left(x^{k}\right) J_{1}$, where $M(\dot{\phi}) f(x)=\phi(x) \cdot f(x)$ (since $\left.T_{1}=J_{1}^{-1} M J_{1}\right)$. So

$$
T_{1}^{k}(x)=x^{k} f(x)+k x^{k-1} \int_{0}^{x} f(t) d t, f \in L^{p}(0,1) .
$$

The linear $\operatorname{span}\left\{x^{k-1}, x^{2 k-1}, x^{3 k-1}, \cdots\right\}$ is dense in $L^{p}(0,1)$, therefore the linear span of $\left\{x^{k}, x^{2 k}, x^{3 k}, \cdots\right\}$ is dense in the range of $\mathscr{R}_{1}$ of $J_{1}$. Thus

$\mathscr{S}$ is a closed $T_{1}^{k}$-invariant subspace $\Leftrightarrow J_{1} \mathscr{S}$ is a closed ideal of $\mathscr{R}_{1}$.

Hence all the operators in $\left\{T_{1}^{k}\right\}_{k=1}^{\infty}$ have exactly the same closed invariant subspaces. 
The set Lat $T_{n}$ of all the closed invariant subspaces of $T_{n}$ is a complete lattice under $\leqq$ where $\mathscr{S}_{1} \leqq \mathscr{S}_{2}$ if $\mathscr{S}_{1} \leqq \mathscr{S}_{2}$. Theorem 3.19 puts $\mathscr{C}^{n}$ in a one-to-one correspondence with the collection of all $n$-tuples $\left\{\left(E_{0}, E_{1}, \cdots, E_{n-1}\right): E_{j}\right.$ 's are closed subsets of $[0,1]$ such that $E_{0} \supseteq E_{1} \supseteq \cdots \supseteq E_{n-1} \supseteq \operatorname{deriv} E_{0}$. Define an ordering on $\mathscr{C}^{n}$ by $\left(E_{0}, E_{1}, \cdots, E_{n-1}\right)<\left(F_{0}, F_{1}, \cdots, F_{n-1}\right)$ if $E_{j} \supseteqq F_{j}, 0 \leqq j \leqq n-1$. This makes $\left(\mathscr{C}^{n},<\right)$ into a lattice.

To show completeness of the lattice, consider any subset of $\mathscr{C}^{n}$, $\left\{\left(E_{0}^{\alpha}, E_{1}^{\alpha}, \cdots, E_{n-1}^{\alpha}\right): \alpha \in I\right.$ an index set $\}$. It is clear that $\mathrm{V}_{\alpha}\left\{\left(E_{0}^{\alpha}, E_{1}^{\alpha}, \cdots\right.\right.$, $\left.\left.E_{n-1}^{\alpha}\right)\right\}=\left(\bigcap_{\alpha} E_{0}^{\alpha}, \cdots, \bigcap_{\alpha} E_{n-1}^{\alpha}\right) \in \mathscr{C}^{n}$, and since the smallest element $([0,1], \cdots,[0,1]) \in \mathscr{C}^{n},\left(\mathscr{C}^{n},<, \wedge, \vee\right)$ is a complete lattice $([2]$, p. 49). However, it can be checked that

$$
\begin{aligned}
\bigwedge_{\alpha} & \left\{\left(E_{0}^{\alpha}, E_{1}^{\alpha}, \cdots, E_{n-1}^{\alpha}\right)\right\} \\
& =\left(\overline{\bigcup_{\alpha} E_{0}^{\alpha}}, \overline{\bigcup_{\alpha} E_{1}^{\alpha}} \cup \operatorname{deriv}\left(\overline{\bigcup_{\alpha} E_{0}^{\alpha}}\right), \cdots, \overline{\bigcup_{\alpha} E_{n-1}^{\alpha}} \cup \operatorname{deriv}\left(\overline{\bigcup_{\alpha} E_{0}^{\alpha}}\right)\right) .
\end{aligned}
$$

Similarly the subcollection $\mathscr{C}_{0}^{n}$ is also a complete sublattice of $\mathscr{C}^{n}$.

THEOREM 4.3. The map $J_{n}$ induces a complete lattice isomorphism w: Lat $T_{n} \rightarrow \mathscr{C}_{0}^{n}$ which is defined as

$$
\psi(\mathscr{S})=\left(Z_{0}(\tilde{\mathscr{S}}), Z_{1}(\tilde{\mathscr{S}}), \cdots, Z_{n-1}(\tilde{\mathscr{S}})\right),
$$

where $\mathscr{S} \in$ Lat $T_{n}$ and $\tilde{\mathscr{S}}=J_{n}(\mathscr{S})$.

The proof is left to the reader.

5. The operators $M \pm \alpha J_{1}$ and $M \pm \alpha J_{1}^{*}$ with $\operatorname{Re} \alpha=n$. We extend our results on $T_{n}$ to the operator $U_{n}: L^{p}(0,1) \rightarrow L^{p}(0,1)$ where

$$
U_{n} f(x)=x f(x)-n \int_{0}^{x} f(t) d t, f \in L^{p}(0,1), n \in N,
$$

and their adjoint operators $T_{n}^{*}$ and $U_{n}^{*}$ on $L^{q}(0,1)(1 / p+1 / q=1)$ where

$$
\begin{aligned}
& T_{n}^{*} f(x)=x f(x)+n \int_{x}^{1} f(t) d t, \quad f \in L^{q}(0,1), \\
& U_{n}^{*} f(x)=x f(x)-n \int_{x}^{1} f(t) d t, \quad f \in L^{q}(0,1) .
\end{aligned}
$$

Furthermore, the parameter can be allowed to be complex with integral real part.

Let us first deal with the operator $U_{n}^{*}$. We shall work with $1 \leqq q<\infty$ instead of $1<q \leqq \infty$.

Define an operator $W_{n}$ on $L^{q}(0,1)$, which is analogous to $J_{n}$, as 


$$
W_{n} f(x)=\frac{1}{\Gamma(n)} \int_{x}^{1}(x-t)^{n-1} f(t) d t, f \in L^{q}(0,1) .
$$

The same argument used in $\$ 3$ can be applied with slight modification and we have the following result which is analogous to Theorems 4.1 and 4.3 .

THEOREM 5.1. $(1 \leqq q<\infty)$. Via the similarity relation $W_{n}^{-1} M W_{n}=$ $U_{n}^{*}$, there is a complete lattice isomorphism between Lat $\left(U_{n}^{*}\right)$ and the lattice of all $n$-tuples $\left(E_{0}, E_{1}, \cdots, E_{n-1}\right)$ of closed subsets of $[0,1]$ where $E_{0} \supseteq E_{1} \supseteq \cdots \supseteqq E_{n-1} \supseteq \operatorname{deriv} E_{0}$ and $1 \in E_{i}, 0 \leqq i \leqq n-1$.

REMARK 5.2. The closed invariant subspaces of $T_{n}^{*}$ (respectively $\left.U_{n}\right)$ on $L^{p}(0,1)(1<p<\infty)$ are $\left\{\mathscr{S}^{\perp}: \mathscr{S}\right.$ is a closed invariant subspace of $T_{n}$ (respectively $\left.\left.U_{n}^{*}\right)\right\}$, where

$$
\mathscr{S}^{\perp}=\left\{\phi \in L^{p}(0,1): \int_{0}^{1} \phi(x) f(x) d x=0, \quad \forall f \in \mathscr{S}\right\} .
$$

Now we turn to complex parameters with integral real parts. The resulting operators are not more complex than those we have investigated. In fact the real part of the parameter is the similarity invariant of these operators. Kantorovitz ([10], [11]) and Kalisch ([8], [9]) had investigated the similarity invariants of the operators $M+\alpha J_{1}$ and $M+\alpha J_{1}^{*}$ where $\alpha \in C$. We shall quote their result.

TheOREM 5.3. For $\beta, \gamma \in \boldsymbol{R}$ (the real numbers), the operators $M+\beta J_{1}$ and $M+(\beta+i \gamma) J_{1}$ (respectively $M+\beta J_{1}^{*}$ and $\left.M+(\beta+i \gamma) J_{1}^{*}\right)$ acting on $L^{p}(0,1)(1<p<\infty)$ are similar.

Through Theorems 4.1, 5.1,5.3 and Remark 5.2, we have now obtained a complete characterization of all closed invariant subspaces for the operators $M \pm \alpha J_{1}$ and $M \pm \alpha J_{1}^{*}$ in the spaces $L^{p}(0,1), 1<$ $p<\infty$, for those complex values of $\alpha$ where $\operatorname{Re} \alpha$ is a positive integer.

6. The case of the parameter with nonintegral real part. When the parameter has nonintegral rerl part, the functions in the range $\mathscr{R}_{\alpha}$ of $J_{\alpha}$ cannot be easily identified. Using results on functional calculus, we establish that $\mathscr{R}_{\alpha}$, for $\operatorname{Re} \alpha \geqq 1$, is an algebra without unit, and that it can indeed be embedded as a closed ideal of a Silov algebra with unit in the same manner as have done for $\mathscr{R}_{n}$.

For $n<\alpha<n+1, n \in N$, it is clear that $x^{j} \notin \mathscr{R}_{\alpha}$ for $j \leqq n-1$, but the situation of $x^{n}$ is governed by the values of $p$ of the $L^{p}$ spaces. 
Proposition 6.1. For $n<\alpha<n+1, n \in N$,

(a) if $1 \leqq p<1 /(\alpha-n)$, then $x^{n} \in \mathscr{R}_{\alpha}$,

(b) if $p \geqq 1 /(\alpha-n)$, then $x^{n} \notin \mathscr{R}_{\alpha}$.

Proof. Observe that $J_{\alpha} x^{-(\alpha-n)}=c x^{n}$, for some constant $c$.

(a) is trivial since $x^{-(\alpha-n)} \in L^{p}(0,1)$.

When $p \geqq 1 /(\alpha-n)$, then $x^{-(\alpha-n)} \notin L^{p}(0,1)$, but for any $q<1 /(\alpha-n)$, $x^{-(\alpha-n)} \in L^{q}(0,1)$. Hence the fact that $J_{\alpha}: L^{q}(0,1) \rightarrow L^{q}(0,1)$ is injective implies that $x^{n} \notin \mathscr{R}_{\alpha}$.

We shall now define $\tilde{\mathscr{R}}_{\alpha}$ accordingly: If $1 \leqq p<1 /(\alpha-n)$, define $\tilde{\mathscr{R}}_{\alpha}=\mathscr{R}_{\alpha} \oplus \boldsymbol{C} x^{n-1} \oplus \boldsymbol{C} x^{n-2} \oplus \cdots \oplus \boldsymbol{C}$; for $p \geqq 1 /(\alpha-n)$, define $\tilde{\mathscr{R}}_{\alpha}=$ $\mathscr{R}_{\alpha} \oplus \boldsymbol{C} x^{n} \oplus \boldsymbol{C} x^{n-1} \oplus \cdots \oplus \boldsymbol{C}$. We will show that for some natural norm, $\tilde{\mathscr{R}}_{\alpha}$ is a Silov algebra with $\mathscr{R}_{\alpha}$ as a closed ideal.

There are some known properties of $T_{\alpha}$ and the Riemann Liouville holomorphic semigroups $\left\{J_{\alpha}: \alpha \in C\right.$ and $\left.\operatorname{Re} \alpha>0\right\}$ which are useful to us. We shall quote them without proof and refer the reader to Hille and Phillips [6], Kalisch [8], [9] and Kantorovitz [10], [11].

Recall that $J_{\alpha} f(x)=1 / \Gamma(\alpha) \int_{0}^{x}(x-t)^{\alpha-1} f(t) d t, f \in L^{p}(0,1)$. These operators are injective and bounded on $L^{p}(0,1)(1 \leqq p<\infty)$ with $\left\|J_{\alpha}\right\| \leqq 1 /(\beta|\Gamma(\alpha)|)$, where $\alpha=\beta+i \gamma, \gamma \in \boldsymbol{R}$ and $\beta>0$. The inverse $J_{\alpha}^{-1}$ (which will also be denoted by $J_{-\alpha}$ ), with domain $\mathscr{R}_{\alpha}$, is thus a closed operator. We also have the identity $(d / d x) J_{\alpha+1} f(x)=J_{\alpha} f(x)$. $\mathscr{R}_{\alpha}$ is invariant under $M$; more precisely, $M J_{\alpha}=J_{\alpha} T_{\alpha}$.

For $1<p<\infty$, the semigroup $\left\{J_{\alpha}: \alpha \in C\right.$. $\left.\operatorname{Re} \alpha>0\right\}$ admits a boundary group of bounded operators $\left\{J_{i r}: \gamma \in \boldsymbol{R}\right\}$ on $L^{p}(0,1)$ with purely imaginary parameters and $\left\|J_{i r}\right\| \leqq e^{\pi|r| / 2}$. For $\beta, \gamma \in \boldsymbol{R}$, $J_{i r} T_{\beta+i r} J_{-i r}=T_{\beta}$; and for $\beta>0, J_{\beta+i r}=J_{\beta} J_{i r}$ which implies that $\mathscr{R}_{\beta+i T}=\mathscr{R}_{\beta}$.

In the papers of Kantorovitz [10], [11], it was shown that the operator $T_{\alpha}$ is of class $C^{n}$ if and only if $|\operatorname{Re} \alpha| \leqq n$. We say that $T_{\alpha}$ is of class $C^{n}$ if there is a continuous representation $\tau: C^{n}[0,1] \rightarrow$ $\mathscr{B}\left(L^{p}(0,1)\right)$ where $\mathscr{B}\left(L^{p}(0,1)\right)$ is the set of bounded operators in $L^{p}(0,1)$, such that $\tau(1)=I$ (the identity operator) for the function $1(t)=1, t \in[0,1]$, and $\tau(x)=T_{\alpha}$. Imitating the argument of Kantorovitz for the $C^{n}$-functional calculus, we shall establish the $\tilde{\mathscr{R}}_{n}$ functional calculus for $T_{\alpha}$, denoting the map by $\tau_{\alpha, n}$. (When there is no confusion, we shall just abbreviate $\tau_{\alpha, n}$ by $\tau$ ). We will then use this $\tilde{\mathscr{R}}_{n}$-functional calculus to prove that $\widetilde{\mathscr{R}}_{\alpha}$ is a Banach algebra under a norm $|\cdot|_{\alpha}$ which is a natural generalization of the norm $|\cdot|_{n}$ of $\widetilde{\mathscr{R}}_{n}$.

For $1 \leqq p<\infty, n \in N, T_{n}$ has $\tilde{\mathscr{P}}_{n}$-functional calculus.

Proposition 6.2. The map $\tau: \tilde{\mathscr{R}}_{n} \rightarrow \mathscr{B}\left(L^{p}(0,1)\right)$ defined by $\tau(\phi)=$ 
$J_{n}^{-1} M(\phi) J_{n}, \phi \in \widetilde{\mathscr{R}}_{n}$, is a continuous representation such that $\tau(1)=I$ and $\tau(x)=T_{n}$, where $M(\phi)$ is the multiplication operator on $L^{p}(0,1)$ by $\phi$.

Proof. That $\tau$ is a representation is trivial.

$$
\begin{aligned}
& \tau(\phi)=\sum_{j=0}^{n}\left(\begin{array}{l}
n \\
j
\end{array}\right) M\left(\phi^{(j)}\right) J_{j} \quad \text { (by Leibnitz's rule), and therefore } \\
& \|\tau(\phi) f\|_{p} \leqq c\|f\|_{p}|\dot{\phi}|_{n},
\end{aligned}
$$

where $\left(\begin{array}{c}n \\ j\end{array}\right)=n ! / j !(n-j) !$ and $c$ is some positive constant depending only on $n$. Thus $\tau$ is continuous and $\mathscr{R}_{n}$-functional calculus is established.

REMARK 6.3. It is trivial but useful to note that the operator $T_{0}$ which is just $M$ also has an $\widetilde{\mathscr{R}}_{n}$-functional calculus for $n \in N, 1 \leqq$ $p<\infty$, namely $\tau(\phi) f=\phi \cdot f, \phi \in \widetilde{\mathscr{R}}_{n}, f \in L^{p}(0,1)$.

The next lemma is an imitation of an argument of Kantorovitz [10].

LEMMA 6.4. $(1<p<\infty)$. Suppose that for some integer $n \geqq 1$ and some $\alpha_{0} \in C, \operatorname{Re} \alpha_{0} \geqq 0, T_{\alpha_{0}}$ is of class $\tilde{\mathscr{R}}_{n}(1<p<\infty)$, then $T_{\alpha}$ is of class $\tilde{\mathscr{R}}_{n}$ for all $\alpha$ in the strip $0 \leqq \operatorname{Re} \alpha \leqq \operatorname{Re} \alpha_{0}$.

Proof. Let $\operatorname{Re} \alpha_{0}=\beta_{0} \geqq 0$ and $\alpha=\beta+i \gamma, \beta, \gamma \in \boldsymbol{R}$. For any fixed polynomial $\phi$ and vectors $f \in L^{p}(0,1), g \in L^{q}(0,1)(1 / p+1 / q=1)$, define

$$
\Phi(\alpha)=\left\langle e^{\pi \alpha^{2}} \phi\left(T_{\alpha}\right) f, g\right\rangle, \quad \alpha \in C,
$$

where $\phi\left(T_{\alpha}\right)$ is the polynomial $\phi$ in $T_{\alpha}$. Observe that for $\operatorname{Re} \alpha \leqq$ $\operatorname{Re} \alpha_{0}=\beta_{0},\left\|T_{\alpha}\right\| \leqq 1+\left(\beta_{0}^{2}+\gamma^{2}\right)^{1 / 2}$ and $\left|e^{\pi \alpha^{2}}\right| \leqq e^{\pi \beta_{0}^{2}}$. Since $\phi\left(T_{\alpha}\right)$ is a polynomial in $\alpha$, with operator coefficients, we thus have for any $\varepsilon>0$,

$$
|\Phi(\alpha)|=0\left(e^{\varepsilon|r|}\right) \text { as }|\gamma| \longrightarrow \infty
$$

in the strip $0 \leqq \operatorname{Re} \alpha \leqq \beta_{0}$. Furthermore,

$$
\left\|e^{\pi \alpha^{2}} \cdot \phi\left(T_{\alpha}\right) f\right\|_{p}=\left\|e^{\pi \alpha^{2}} \cdot\left(J_{-i \tau} \phi\left(T_{\beta}\right) J_{i \curlyvee}\right) f\right\|_{p},
$$

therefore

$$
\begin{aligned}
|\Phi(\alpha)| & \leqq e^{\pi\left(\beta^{2}-\gamma^{2}+|\gamma|\right)}\|f\|_{p}\|g\|_{p}\left\|\phi\left(T_{\beta}\right)\right\| \\
& \leqq e^{\pi\left(\beta^{2}+1 / 4\right)}\|f\|_{p}\|g\|_{q}\left\|\phi\left(T_{\beta}\right)\right\| .
\end{aligned}
$$

Now $T_{0}$ and $T_{\beta_{0}}$ are of class $\tilde{\mathscr{R}}_{n}$, so there is some constant $K>0$ 
such that

$$
\left\|\phi\left(T_{0}\right)\right\| \leqq K|\phi|_{n} \quad \text { and } \quad\left\|\phi\left(T_{\beta_{0}}\right)\right\| \leqq K|\phi|_{n}
$$

Thus

$$
\begin{aligned}
& |\Phi(0+i \gamma)| \leqq A\|f\|_{p}\|g\|_{q}|\dot{\phi}|_{n} \\
& \left|\Phi\left(\beta_{0}+i \gamma\right)\right| \leqq A\|f\|_{p}\|g\|_{q}|\phi|_{n},
\end{aligned}
$$

where $A=K e^{\left(\beta_{0}^{2}+1 / 4\right)}$. By the Paragman-Lindelof Principle ([22], p. 180),

$$
|\Phi(\alpha)| \leqq A\|f\|_{p}\|g\|_{p}|\phi|_{n}, \quad \text { for all } 0 \leqq \operatorname{Re} \alpha \leqq \beta_{0} .
$$

Therefore for $0 \leqq \operatorname{Re} \alpha \leqq \beta_{0}$,

$$
\left\|\phi\left(T_{\alpha}\right)\right\| \leqq A e^{\pi\left(\gamma^{2}-\beta^{2}\right)}|\phi|_{n} .
$$

Since the polynomials are dense in $\tilde{\mathscr{R}}_{n}$, the homomorphism $\phi \rightarrow \phi\left(T_{\alpha}\right)$ can be extended continuously to a homomorphism $\tau$ on $\widetilde{\mathscr{R}}_{n}$. Thus $T_{\alpha}$ is class $\tilde{\mathscr{R}}_{n}$.

An immediate consequence of Proposition 6.2 and Lemma 6.4 is:

CoRollary 6.5. For $n \in N, 0 \leqq \operatorname{Re} \alpha \leqq n(1<p<\infty), T_{\alpha}$ has $\tilde{\mathscr{R}}_{n}$-functional calculus.

The $\tilde{\mathscr{R}}_{n}$-functional calculus of $T_{\alpha}$ is explicitly determined below.

Proposition 6.6. $(1<p<\infty)$. For $0 \leqq \operatorname{Re} \alpha \leqq n, \mathscr{R}_{\alpha}$ is invariant under $M(\phi)$, where $\phi \in \widetilde{\mathscr{R}}_{n}$, and the $\tilde{\mathscr{R}}_{n}$-functional calculus $\tau: \widetilde{\mathscr{R}}_{n} \rightarrow$ $\mathscr{B}\left(L^{p}(0,1)\right.$ for $T_{\alpha}$ is given by

$$
\tau(\phi)=J_{-\alpha} M(\phi) J_{\alpha}, \phi \in \tilde{\mathscr{R}}_{n} .
$$

Proof. First let $\phi$ be any polynomial. Clearly $M(\phi) J_{\alpha}=J_{\alpha} \phi\left(T_{\alpha}\right)$; therefore $\phi\left(T_{\alpha}\right)=J_{-\alpha} M(\phi) J_{\alpha}$. Since $\phi$ is a polynomial and $\tau$ defines the functional calculus, $\tau(\phi)=\phi\left(T_{\alpha}\right)$, and hence $\tau(\phi)=J_{-\alpha} M(\phi) J_{\alpha}$.

Now let $\phi \in \mathscr{R}_{n}$. There exists polynomials $\left\{\phi_{k}\right\}$ such that $\phi_{k} \rightarrow \dot{\phi}$ in $\mathscr{R}_{n}$; in particular, $\phi_{k} \rightarrow \phi$ uniformly in $[0,1]$. Therefore $\tau\left(\phi_{k}\right) \rightarrow$ $\tau(\phi)$ and for any $g \in L^{p}(0,1)$,

$$
\begin{gathered}
\phi_{k} \cdot J_{\alpha} g \longrightarrow \phi \cdot J_{\alpha} g \quad \text { in } L^{p}(0,1), \\
J_{-\alpha} \phi_{k} J_{\alpha} g=\phi_{k}\left(T_{\alpha}\right) g \longrightarrow \tau(\phi) g \text { in } L^{p}(0,1) .
\end{gathered}
$$

The graph of $J_{-\alpha}$ is closed in $\mathscr{R}_{\alpha} \times L^{p}(0,1)$. Thus (1) and (2) imply that $\phi \cdot J_{\alpha} g \in \mathscr{R}_{\alpha}$ and $J_{-\alpha} \phi \cdot J_{\alpha} g=\tau(\phi) g$.

This proves that $\tau(\phi)=J_{-\alpha} M(\phi) J_{\alpha}$, for all $\phi \in \tilde{\mathscr{R}}_{n}$. 
COROLLARY 6.7. For $\operatorname{Re} \alpha \geqq 1(1<p<\infty) \widetilde{\mathscr{R}_{\alpha}}$ is an algebra.

Proof. Without loss of generality, we may assume $\alpha$ to be real, since $\mathscr{R}_{\alpha}=\mathscr{R}_{\text {Re } \alpha}$. It suffices to consider only non-integral values of $\alpha$ and we only need to show that $\mathscr{R}_{\alpha}$ is closed under multiplication, since $\mathscr{R}_{\alpha}$ is invariant under $M$.

Write $\alpha=n+r$ where $n$ is an integer $\geqq 1,0<r<1$. Let $f, g \in$ $L^{p}(\mathbf{0}, \mathbf{1})$.

$$
J_{n}^{-1}\left(J_{\alpha}(f) \cdot J_{\alpha}(g)\right)=\sum_{j=0}^{n}\left(\begin{array}{c}
n \\
j
\end{array}\right) J_{\alpha-j}(f) J_{\alpha-(n-j)}(g) .
$$

Proposition 6.6 implies that for $0 \leqq j \leqq n$,

$$
J_{\alpha-j}(f) \cdot J_{\alpha-(n-j)}(g) \in J_{r}\left(L^{p}(0,1)\right) .
$$

Thus $J_{\alpha}(f) \cdot J_{\alpha}(g) \in J_{n+r}\left(L^{p}(0,1)\right)=J_{\alpha}\left(L^{p}(0,1)\right)$ and hence the result.

On $\mathscr{R}_{\alpha}, \operatorname{Re} \alpha>0$, let us define $\left|J_{\alpha} f\right|_{\alpha}=\|f\|_{p}, f \in L_{p}(0,1)$. Then $J_{\alpha}$ is an isometry from $L^{p}(0,1)$ onto $\mathscr{R}_{\alpha}$.

Proposition 6.8. $(1<p<\infty)$. For $\operatorname{Re} \alpha \geqq 1$, there exists $a$ constant $c_{\alpha}>0$ such that for all $f, g \in L^{p}(0,1)$,

$$
\left|J_{\alpha}(f) \cdot J_{\alpha}(g)\right|_{\alpha} \leqq c_{\alpha}\left|J_{\alpha}(f)\right|_{\alpha}\left|J_{\alpha}(g)\right|_{\alpha} \text {. }
$$

Proof. We need to consider only real nonintegral $\alpha$. Write $\alpha=n+r$ where $n$ is an integer $\geqq 1$ and $0<r<1$. By Leibnitz's rule,

$$
J_{n}^{-1}\left(J_{n+r}(f) \cdot J_{n+r}(g)\right)=\sum_{j=0}^{n}\left(\begin{array}{c}
n \\
j
\end{array}\right) J_{n+r-j}(f) J_{n+r-(n-j)}(g)
$$

Therefore $\left|J_{n+r}(f) \cdot J_{n+r}(g)\right|_{\alpha} \leqq \sum_{j=0}^{n}\left(\begin{array}{c}n \\ j\end{array}\right)\left|J_{n+r-j}(f) \cdot J_{r+j}(g)\right|_{r}$. For $1 \leqq$ $j \leqq n$, by Proposition 6.6,

$$
J_{r+j}(g) \cdot J_{r}\left(J_{n-j}(f)\right)=J_{r}\left(\tau_{r, 1}\left(J_{r+j}(g)\right)\right)\left(J_{n-j}(f)\right) .
$$

Now

$$
\left|J_{r+j}(g)\right|_{1} \leqq \frac{1}{\Gamma(r+j)}\|g\|_{p}
$$

therefore

$$
\begin{aligned}
\left|J_{n+r-j}(f) \cdot J_{r+j}(g)\right|_{r} & =\left\|\left(\tau_{r, 1}\left(J_{r+j}(g)\right)\right) J_{n-j}(f)\right\|_{p} \\
& \leqq\left\|\tau_{r, 1}\right\| \frac{1}{\Gamma(r+j)}\|g\|_{p} \frac{1}{(n-j) !}\|f\|_{p} .
\end{aligned}
$$

Similarly there exists a constant $K_{\alpha}>0$, dependent only on $\alpha$, such that 


$$
\left|J_{n+r}(f) \cdot J_{r}(g)\right| \leqq K_{\alpha}\|f\|_{p}\|g\|_{p} .
$$

Thus there exists a constant $c_{\alpha}>0$ which depends only on $\alpha$, such that

$$
\left|J_{n+r}(f) \cdot J_{n+r}(g)\right|_{\alpha} \leqq c_{\alpha}\left|J_{n+r}(f)\right|_{\alpha}\left|J_{n+r}(g)\right|_{\alpha} \text {, for all } f, g \in L^{p}(0,1) \text {. }
$$

Now we can norm $\widetilde{\mathscr{R}}_{\alpha}$ a Banach algebra. Our attention will be restricted to the case when $1 \leqq p<1 /(\alpha-n)$ since the same argument holds for the complementary case.

Every element $F$ of $\tilde{\mathscr{R}}_{\alpha}$ is of the form $J_{\alpha} f+\sum_{i=0}^{n-1} c_{i} x_{i}, f \in L^{p}(0,1)$, $c_{i} \in C$. We extend the norm $|\cdot|_{\alpha}$ on $\mathscr{R}_{\alpha}$ to $\tilde{\mathscr{R}}_{\alpha}$, namely,

$$
|F|_{\alpha}=\|f\|_{p}+\sum_{n=0}^{n-1}\left|c_{i}\right| \text {, }
$$

then $\widetilde{\mathscr{R}}_{\alpha}$ is a Banach space with $\mathscr{R}_{\alpha}$ as a closed ideal. Furthermore, $|\cdot|_{\alpha}$ is equivalent to some Banach algebra norm, i.e., for any $F, G \in$ $\widetilde{\mathscr{R}}_{\alpha}$, there exists a constant $K_{\alpha}$, dependent only on $\alpha$ and $p$, such that

$$
|F G|_{\alpha} \leqq K_{\alpha}|F|_{\alpha}|G|_{\alpha} .
$$

The map $F \rightarrow F^{(j)}(a), 0 \leqq j \leqq \alpha-1$, is continuous on $\tilde{\mathscr{R}}_{\alpha}$.

As it would have been expected, the maximal ideal space of $\widetilde{\mathscr{R}}_{\alpha}$ is $[0,1]$ and it is then evident that $\tilde{\mathscr{R}}_{\alpha}$ is semisimple. With $C^{\infty}$ being contained in $\widetilde{\mathscr{R}}_{\alpha}$, it is clear that $\widetilde{\mathscr{R}}_{\alpha}$ a Silov algebra.

By carrying out the same argument used previously for $\tilde{\mathscr{R}}_{n}$ functional calculus, we can establish the $\tilde{\mathscr{R}}_{\alpha}$-functional calculus, $\operatorname{Re} \alpha \geqq 1$.

TheOREM 6.10. $(1<p<\infty)$. For $\alpha, \beta \in C, \operatorname{Re} \alpha \geqq 1$ and $0 \leqq \operatorname{Re} \beta \leqq$ $\operatorname{Re} \alpha, T_{\beta}$ has $\tilde{\mathscr{R}}_{\alpha}$-functional calculus $\tau: \tilde{\mathscr{R}}_{\alpha} \rightarrow \mathscr{\mathscr { B }}\left(L^{p}(0,1)\right)$,

$$
\tau(\phi)=J_{-\beta} M(\phi) J_{\beta}, \phi \in \widetilde{\mathscr{R}}_{\alpha} .
$$

We conclude our discussion with the following remark.

REMARK 6.11. By virtue of the identity $J_{-\alpha} M J_{\alpha}=T_{\alpha}$ and the fact that the polynomials are dense in $\mathscr{R}_{\alpha}, \operatorname{Re} \alpha>1$, the closed $T_{\alpha^{-}}$ invariant subspaces are in one-to-one correspondence with the closed ideals of $\mathscr{R}_{\alpha}$ which are closed under multiplication by the function $x$.

\section{REFERENCES}

1. A. Beurling, On two problems concerning linear transformations in Hilbert space, Acta Math., 89 (1949), 249-255.

2. G. Birhoff, Latthce Theory, Amer. Math. Soc., 1948. 
3. J. T. Daly and P. B. Downum, A Banach algebra of functions with bounded $n$th differences, Trans. Amer. Math. Soc., 223 (1976), 279-294.

4. W. F. Donoghue, The lattice of invariant subspaces of a completely continuous quasi. nilpotent transformation, Pacific J. Math., 7 (1957), 1031-1035.

5. J. A. Erdös, On a certain abelian algebra of operators and their invariant subspace lattices, Proc. London Math. Soc., (3) 29 (1974), 77-97.

6. E. Hille and R. S. Phillips, Functional Analysis and Semi-groups, Amer. Math. Soc., Colloq. Publ., Vol. 31, Amer. Math. Soc., Providence, R.I., 1957.

7. G. K. Kalisch, On similarity, reducing manifolds, and unitary equivalence of certain Volterra operators, Ann. of Math., 66 (1957), 481-494.

8. - On fractional integrals of pure imaginary order in $L^{p}$, Proc. Amer. Math. Soc., 18 (1967), 136-139.

9. - On the similarity of certain operators, Colloquia Mathematica Societatis Jànos Bolyai, 5, Hilbert Space Operators, Tihany (Hungary), 1970.

10. S. Kantorovitz, The $C^{k}$-classification of certain operators in $L^{p}$, Trans. Amer. Math. Soc., 132 (1968), 323-333.

11. The $C^{k}$-classification of certain operators in $L^{p}, I I$, Trans. Amer. Math. Soc., 146 (1969), 61-67.

12. B. I. Korenbljum, Invariant subspaces of the shift operator in weighted Hilbert space, Mat. Sbornik, 89 (131) (1972), 110-137=Math. USSR Sbornik, 18 (1972), 111-138. 13. M. A. Naimark, Normed Albebras, Wolters-Noordhoff Publishing, Groningen, The Netherlands, 1972.

14. N. K. Nikol'skii, Invariant subspaces of certain completely continuous operators, Vestnik Leningrad. Univ., 20 (1965), No. 7, 68-77 (Russian).

15. - Invariant subspaces of weighted shift operators, Mat. Sbornik, 74 (116) (1967), 171-190 = Math. USSR Sbornik, 3 (1967), 159-176.

16. N. M. Osadchii, The $L_{n}^{2}(\Gamma)$ algebras and the structures of their closed ideals, Ukr. Mat. Zh. 26, No. 5 (1974), 669-670 = Ukr. Math. J., (1975), 548-549.

17. H. Radjavi and P. Rosenthal, Invariant Subspaces, Springer-Verlag, Berlin-Heidelberg-New York, 1973.

18. D. Sarason, Topics in Operator Theory, Mathematical Surveys, No. 13, C. Pearcy, ed., Amer. Math. Soc., Providence, R.I., 1974, 3-47.

19. G. E. Silov, On regular normed rings, Trav. Inst. Math. Stekloff, 21 (1947), 118 pp.

20. I. E. Snol', The structure of ideals in rings $R_{\alpha}$, Mat. Sbornik, (N.S.) 27 (69) (1950), 143-146 (Russian).

21. M. H. Stone, Applications of the theory of Boolean rings to general topology, Trans. Amer. Math. Soc., 41 (1937), 375-481.

22. E. C. Titchmarsh, The Theory of Functions, Oxford Univ. Press, London, 1939.

23. R. E. Waterman, Invariant subspaces, similarity and isometric equivalence of certain commuting operators in $L^{p}$, Pacific J. Math., 48 No. 2 (1973), 593-613.

24. H. Whitney, On ideals of differentiable functions, Amer. J. Math., 70 (1948), 635-658.

Received August 9, 1980. This paper is condensed from the author's Ph. D. dissertation at University of California, Berkeley, June 1978. The author wishes to express her deep gratitude to her thesis adviser Professor W. G. Badé and Professor D. Sarason.

University of Science of Malaysia

Penang, Malaysia 



\section{PACIFIC JOURNAL OF MATHEMATICS}

\section{EDITORS}

DONALD BABBITT (Managing Editor)

University of California

Los Angeles, CA 90024

Hugo RossI

University of Utah

Salt Lake City, UT 84112

C. C. MOORE and ANDREW OGG

University of California

Berkeley, CA 94720
J. DugundjI

Department of Mathematics

University of Southern California

Los Angeles, CA 90007

R. FINN and J. MILGRAM

Stanford University

Stanford, CA 94305

\section{ASSOCIATE EDITORS}
R. ARENS
E. F. BECKENBACH
B. H. NeUmanN
F. WOLF
K. YosHIDA

\section{SUPPORTING INSTITUTIONS}

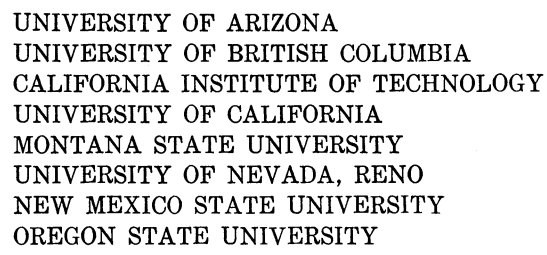

UNIVERSITY OF ARIZONA

UNIVERSITY OF BRITISH COLUMBIA CALIFORNIA INSTITUTE OF TECHNOLOGY

UNIVERSITY OF CALIFORNIA

MONTANA STATE UNIVERSITY

UNIVERSITY OF NEVADA, RENO

NEW MEXICO STATE UNIVERSITY OREGON STATE UNIVERSITY

\author{
UNIVERSITY OF OREGON \\ UNIVERSITY OF SOUTHERN CALIFORNIA \\ STANFORD UNIVERSITY \\ UNIVERSITY OF HAWAII \\ UNIVERSITY OF TOKYO \\ UNIVERSITY OF UTAH \\ WASHINGTON STATE UNIVERSITY \\ UNIVERSITY OF WASHINGTON
}

The Supporting Institutions listed above contribute to the cost of publication of this Journal, but they are not owners or publishers and have no responsibility for its content or policies.

Mathematical papers intended for publication in the Pacific Journal of Mathematics should be in typed form or offset-reproduced, (not dittoed), double spaced with large margins. Please do not use built up fractions in the text of the manuscript. However, you may use them in the displayed equations. Underline Greek letters in red, German in green, and script in blue. The first paragraph or two must be capable of being used separately as a synopsis of the entire paper. Please propose a heading for the odd numbered pages of less than 35 characters. Manuscripts, in triplicate, may be sent to any one of the editors. Please classify according to the scheme of Math. Reviews, Index to Vol. 39. Supply name and address of author to whom proofs should be sent. All other communications should be addressed to the managing editor, or Elaine Barth, University of California, Los Angeles, California, 90024.

50 reprints to each author are provided free for each article, only if page charges have been substantially paid. Additional copies may be obtained at cost in multiples of 50 .

The Pacific Journal of Mathematics is issued monthly as of January 1966. Regular subscription rate: $\$ 102.00$ a year (6 Vols., 12 issues). Special rate: $\$ 51.00$ a year to individual members of supporting institutions.

Subscriptions, orders for numbers issued in the last three calendar years, and changes of address shoud be sent to Pacific Journal of Mathematics, P.O. Box 969, Carmel Valley, CA 93924, U.S.A. Old back numbers obtainable from Kraus Per!odicals Co., Route 100, Millwood, NY 10546.

\section{PUBLISHED BY PACIFIC JOURNAL OF MATHEMATICS, A NON-PROFIT CORPORATION}

Printed at Kokusai Bunken Insatsusha (International Academic Printing Co., Ltd.). 8-8, 3-chome, Takadanobaba, Shinjuku-ku, Tokyo 160, Japan.

Copyright (C) 1981 by Pacific Jounal of Mathematics Manufactured and first issued in Japan 


\section{Pacific Journal of Mathematics}

Vol. 94, No. $2 \quad$ June, 1981

Thomas E. Armstrong and William David Sudderth, Nearly strategic

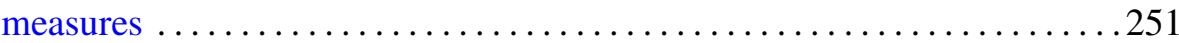

John J. Buoni, Artatrana Dash and Bhushan L. Wadhwa, Joint Browder

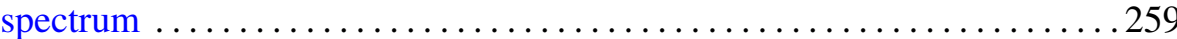

Jack Paul Diamond, Hypergeometric series with a $p$-adic variable . .......265

Raymond Frank Dickman, Jack Ray Porter and Leonard Rubin,

Completely regular absolutes and projective objects ............ 277

James Kenneth Finch, On the local spectrum and the adjoint ........... 297

Benno Fuchssteiner, An abstract disintegration theorem ............ 303

Leon Gerber, The volume cut off a simplex by a half-space $\ldots \ldots \ldots \ldots 311$

Irving Leonard Glicksberg, An application of Wermer's subharmonicity

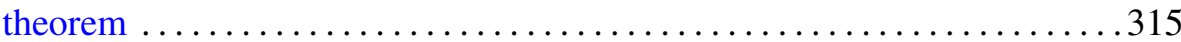

William Goldman, Two examples of affine manifolds ............... 327

Yukio Hirashita, On the Weierstrass points on open Riemann surfaces .....331

Darrell Conley Kent, A note on regular Cauchy spaces ............. 333

Abel Klein and Lawrence J. Landau, Periodic Gaussian

Osterwalder-Schrader positive processes and the two-sided Markov

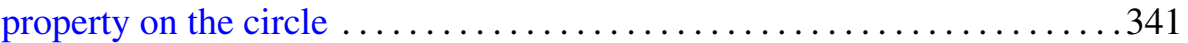

Brenda MacGibbon, $\mathscr{K}$-Borelian embeddings and images of Hausdorff

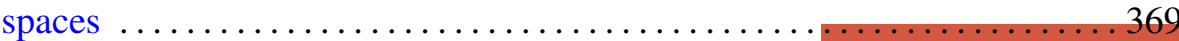

John R. Myers, Homology 3-spheres which admit no PL involutions . . . . . 379

Boon-Hua Ong, Invariant subspace lattices for a class of operators . . . . . 385

Chull Park, Representations of Gaussian processes by Wiener processes . . . 407

Lesley Millman Sibner and Robert Jules Sibner, A sub-elliptic estimate

for a class of invariantly defined elliptic systems $\ldots \ldots \ldots \ldots \ldots \ldots .417$

Justin R. Smith, Complements of codimension-two submanifolds. III.

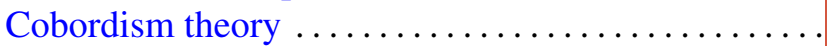

William Albert Roderick Weiss, Small Dowker spaces

David J. Winter, Cartan subalgebras of a Lie algebra and its ideals. II ... 